\title{
Bayesian Analysis of Warner's Randomized Response Technique
}

\author{
A. O. Adepetun ${ }^{1 *}$, A. A. Adewara ${ }^{2}$ \\ ${ }^{1}$ Department of Statistics, Federal University of Technology, PMB 704, Akure, Ondo State, \\ Nigeria \\ ${ }^{2}$ Department of Statistics, University of Ilorin, PMB 1515, Ilorin, Kwara State, Nigeria
}

Received 30 May 2016, accepted in final revised form 30 June 2016

\begin{abstract}
This paper proposed alternative beta estimators of the population proportion of a sensitive attribute when life data were obtained through the administration of survey questionnaires on abortion of some matured women. The results showed that the proposed alternative beta estimators were more efficient in capturing responses from respondents than the simple beta estimator proposed by Winkler and Franklin for relatively small, medium as well as large sample sizes respectively.
\end{abstract}

Keywords: Alternative beta estimators; Simple beta estimator; Sensitive attribute; Mean square error; Absolute bias.

(C) 2017 JSR Publications. ISSN: 2070-0237 (Print); 2070-0245 (Online). All rights reserved. doi: http://dx.doi.org/10.3329/jsr.v9i1.27943 J. Sci. Res. 9 (1), 13-26 (2016)

\section{Introduction}

Asking individuals about sexually-related activity, criminal activity, induced abortion, etc., is an uneasy exercise. Individuals are often reluctant to freely divulge such information in classical survey settings for fear of social disgrace. Yet, for public-health and socio-economic reasons, estimates of the incidence of such activities are essential. The Randomized Response Technique (RRT) can be a useful survey method to find such estimates because individual anonymity is preserved. Initial RRT proposed by Warner [1] and Greenberg et al. [2] presumed that two yes/no questions were provided for each respondent and that a randomization device was used to determine which question would be answered. Since the interviewer would not know the result of the device, participants would be encouraged to give truthful responses to a sensitive question. This technique has generated much interest in the statistical literature since the publication of Warner's

\footnotetext{
Corresponding author: aoadepetun@futa.edu.ng
} 
RRT. A comprehensive literature review will not be provided here. Instead, the interested reader is referred to the review-oriented references listed herein; namely, Mangat et al. [3,4], Christofides [5], Kim and Warde [6], Adebola and Adepetun [7-9]. In some situations, prior information about the unknown parameter may be available and can be combined with the sample information for the estimation of that unknown parameter. This is known as the Bayesian approach of estimation. Work done by researchers on Bayesian Analysis of RRTs are not very extensive, however, attempts have been made on the Bayesian Analysis of RRTs. Winkler and Franklin [10], Pitz [11], O'Hagan [12], Oh [13], Migon and Tachibana [14], Unnikrishnan and Kunte [15], Kim et al. [16], Hussain and Shabbir [17-19], Adepetun and Adewara [20], Adepetun and Adewara [21,22], are the major references on the Bayesian analysis of the RRTs. This paper presents Bayesian analysis to Warner's RRT using alternative beta priors other than the simple beta prior used by Winkler and Franklin [10] in their published paper.

\section{The Conventional Bayesian Technique of Estimation}

Winkler and Franklin [10] in their published paper presented a Bayesian estimation to the RRT put forward by Warner [1] assuming a simple beta prior to estimate the population proportion of respondents possessing sensitive attribute.

Suppose the simple beta prior is defined as follows

$f(\pi)=\frac{1}{B(a, b)} \pi^{a-1}(1-\pi)^{b-1} ; 0<\pi<1$

where $(a, b)$ are the shape parameters of the distribution and $\pi$ is the population proportion of respondents possessing the sensitive attribute.

Let $x=\sum x_{i}$ be the total number of the women who have committed abortion for a particular sample of size $n$ selected from the population with simple random sampling. Then the conditional distribution of $X$ given $\pi$ is

$f(X \mid \pi)=\left(\begin{array}{l}n \\ x\end{array}\right) \phi^{x}(1-\phi)^{n-x}$

Where $\phi$ is the probability of "yes response" to the sensitive attribute which was defined as

$\phi=P($ yes $)=P \pi+(1-P)(1-\pi)$

Where $P$ is the preset probability of "yes" response to the sensitive attribute

$f(X \mid \pi)=\left(\begin{array}{l}n \\ x\end{array}\right)[1-\pi-P+2 P \pi]^{x}[\pi+P-2 P \pi]^{n-x}$

On simplification, we have

$f(X \mid \pi)=\left(\begin{array}{l}n \\ x\end{array}\right)[2 P-1]^{n}[\pi+f]^{x}[1-\pi+h]^{n-x}$

where

$h=f=\frac{1-P}{2 P-1}$ 
Recall from binomial series expansion,

$(\pi+f)^{x}=\sum_{i=0}^{x}\left(\begin{array}{l}x \\ i\end{array}\right) f^{x-i} \pi^{i}$

$(1-\pi+f)^{n-x}=\sum_{j=0}^{n-x}\left(\begin{array}{c}n-x \\ j\end{array}\right) f^{n-x-j}(1-\pi)^{j}$

$f(X \mid \pi)=\left(\begin{array}{l}n \\ x\end{array}\right)[2 P-1]^{n} \sum_{i=0}^{x} \sum_{j=0}^{n-x}\left(\begin{array}{c}x \\ i\end{array}\right)\left(\begin{array}{c}n-x \\ j\end{array}\right) f^{n-i-j} \pi^{i}(1-\pi)^{j}$

for $x=0,1,2, \ldots, n$

Thus, the joint probability density functions (pdf) of $X$ and $\pi$ was

$f(X, \pi)=\frac{\left(\begin{array}{l}n \\ x\end{array}\right)[2 P-1]^{n}}{B(a, b)} \sum_{i=0}^{x} \sum_{j=0}^{n-x}\left(\begin{array}{c}x \\ i\end{array}\right)\left(\begin{array}{c}n-x \\ j\end{array}\right) f^{n-i-j} \pi^{i} \pi^{a-1}(1-\pi)^{b-1}(1-\pi)^{j}$

Now the marginal distribution of $X$ can be obtained by integrating the joint distribution of $X$ and $\pi$ with respect to $\pi$. Thus the marginal distribution of $X$ was given by

$f(X)=\int_{0}^{1} f(X, \pi) d \pi=\frac{[2 P-1]^{n}}{B(a, b)}\left(\begin{array}{l}n \\ x\end{array}\right) \sum_{i=0}^{x} \sum_{j=0}^{n-x}\left(\begin{array}{l}x \\ i\end{array}\right)\left(\begin{array}{c}n-x \\ j\end{array}\right) f^{n-i-j} \int_{0}^{1} \pi^{a+i-1}(1-\pi)^{b+j-1} d \pi$
$f(X)=\frac{[2 P-1]^{n}}{B(a, b)}\left(\begin{array}{l}n \\ x\end{array}\right) \sum_{i=0}^{x} \sum_{j=0}^{n-x}\left(\begin{array}{l}x \\ i\end{array}\right)\left(\begin{array}{c}n-x \\ j\end{array}\right) f^{n-i-j} B(a+i, b+j)$

The posterior distribution of $\pi$ given $X$ was defined as

$$
\begin{aligned}
& f(\pi \mid X)=\frac{f(X, \pi)}{f(X)} \\
& f(\pi \mid X)=\frac{\frac{[2 P-1]^{n}}{B(a, b)}\left(\begin{array}{c}
n \\
x
\end{array}\right) \sum_{i=0}^{x} \sum_{j=0}^{n-x}\left(\begin{array}{c}
x \\
i
\end{array}\right)\left(\begin{array}{c}
n-x \\
j
\end{array}\right) f^{n-i-j} \pi^{a+i-1}(1-\pi)^{b+j-1}}{\frac{[2 P-1]^{n}}{B(a, b)}\left(\begin{array}{c}
n \\
x
\end{array}\right) \sum_{i=0}^{x} \sum_{j=0}^{n-x}\left(\begin{array}{c}
x \\
i
\end{array}\right)\left(\begin{array}{c}
n-x \\
j
\end{array}\right) f^{n-i-j} B(a+i, b+j)} \\
& f(\pi \mid X)=\frac{\sum_{i=0}^{x} \sum_{j=0}^{n-x}\left(\begin{array}{c}
x \\
i
\end{array}\right)\left(\begin{array}{c}
n-x \\
j
\end{array}\right) f^{n-i-j} \pi^{a+i-1}(1-\pi)^{b+j-1}}{\sum_{i=0}^{x} \sum_{j=0}^{n-x}\left(\begin{array}{c}
x \\
i
\end{array}\right)\left(\begin{array}{c}
n-x \\
j
\end{array}\right) f^{n-i-j} B(a+i, b+j)}
\end{aligned}
$$

The Bayes estimator of $\pi$ which is the posterior mean of (8) was given by

$$
\begin{aligned}
& \hat{\pi}_{S W}=\int_{0}^{1} \pi f(\pi \mid X) d \pi=\frac{\sum_{i=0}^{x} \sum_{j=0}^{n-x}\left(\begin{array}{c}
x \\
i
\end{array}\right)\left(\begin{array}{c}
n-x \\
j
\end{array}\right) f^{n-i-j} \int_{0}^{1} \pi^{a+i-1}(1-\pi)^{b+j-1} d \pi}{\sum_{i=0}^{x} \sum_{j=0}^{n-x}\left(\begin{array}{c}
x \\
i
\end{array}\right)\left(\begin{array}{c}
n-x \\
j
\end{array}\right) f^{n-i-j} B(a+i, b+j)} \\
& =\frac{\sum_{i=0}^{x} \sum_{j=0}^{n-x}\left(\begin{array}{c}
x \\
i
\end{array}\right)\left(\begin{array}{c}
n-x \\
j
\end{array}\right) f^{n-i-j} B(a+i+1, b+j)}{\sum_{i=0}^{x} \sum_{j=0}^{n-x}\left(\begin{array}{c}
x \\
i
\end{array}\right)\left(\begin{array}{c}
n-x \\
j
\end{array}\right) f^{n-i-j} B(a+i, b+j)}
\end{aligned}
$$

The Bias of $\hat{\pi}_{S H}$ and its Mean Square Error (MSE) were given by $B\left(\hat{\pi}_{S W}\right)=\hat{\pi}_{S W}-\pi$ 


$$
\operatorname{MSE}\left(\hat{\pi}_{S W}\right)=\sum_{x=0}^{n}\left(\hat{\pi}_{S W}-\pi\right)^{2} \phi^{x}(1-\phi)^{n-x}
$$

\section{The Proposed Bayesian Techniques of Estimation}

In this section, we propose an alternative Bayesian estimation to Warner [1] RRT assuming both the Kumaraswamy and the Generalised beta priors in addition to the simple beta prior proposed by Winkler and Franklin [10].

\subsection{Estimation of $\pi$ using Kumaraswamy prior}

The Kumaraswamy prior distribution of $\pi$ is given as

$$
f(\pi)=b c \pi^{c-1}\left(1-\pi^{c}\right)^{b-1} ; b, c>0
$$

Using the Kumaraswamy prior in (12), the joint probability density function of $X$ and $\pi$ is derived as

$f(X, \pi)=a b A \sum_{i=0}^{x} \sum_{j=0}^{n-x}\left(\begin{array}{l}x \\ i\end{array}\right)\left(\begin{array}{c}n-x \\ j\end{array}\right) f^{x-i} h^{n-x-j} \pi^{i} \pi^{c-1}(1-\pi)^{j}\left(1-\pi^{c}\right)^{b-1}$

where $A=\left(\begin{array}{l}n \\ x\end{array}\right)(2 p-1)^{n}$

The marginal probability density function (pdf) of $X$ can be obtained as

$f(X)=\int_{0}^{1} f(X, \pi) d \pi$

$=a b A \sum_{i=0}^{x} \sum_{j=0}^{n-x} \sum_{k=0}^{b-1}(-1)^{k}\left(\begin{array}{c}x \\ i\end{array}\right)\left(\begin{array}{c}n-x \\ j\end{array}\right)\left(\begin{array}{c}b-1 \\ k\end{array}\right) f^{n-i-j} B(i+c(1+k), j+1)$

$=a b A \sum_{i=0}^{x} \sum_{j=0}^{n-x} \sum_{k=0}^{b-1}(-1)^{k}\left(\begin{array}{l}x \\ i\end{array}\right)\left(\begin{array}{c}n-x \\ j\end{array}\right)\left(\begin{array}{c}b-1 \\ k\end{array}\right) f^{n-i-j} \int_{0}^{1} \pi^{c+i-1+c k}(1-\pi)^{j} d \pi$

Similarly, the posterior distribution as usual is obtained as follows

$$
\begin{gathered}
f(\pi \mid X)=\frac{f(X, \pi)}{f(X)} \\
=\frac{\sum_{i=0}^{x} \sum_{j=0}^{n-x} \sum_{k=0}^{b-1}(-1)^{k}\left(\begin{array}{c}
x \\
i
\end{array}\right)\left(\begin{array}{c}
n-x \\
j
\end{array}\right)\left(\begin{array}{c}
b-1 \\
k
\end{array}\right) f^{n-i-j} \pi^{c+i-1+c k}(1-\pi)^{j}}{\sum_{i=0}^{x} \sum_{j=0}^{n-x} \sum_{k=0}^{b-1}(-1)^{k}\left(\begin{array}{c}
x \\
i
\end{array}\right)\left(\begin{array}{c}
n-x \\
j
\end{array}\right)\left(\begin{array}{c}
b-1 \\
k
\end{array}\right) f^{n-i-j} B(i+c(1+k), j+1)}
\end{gathered}
$$

Under the Square error loss, we proceed to obtain the posterior mean which is the Bayes estimator as follows

$\hat{\pi}_{K W}=\int_{0}^{1} \pi f(\pi \mid X) d \pi$

Therefore,

$\hat{\pi}_{K W}=\frac{\sum_{i=0}^{x} \sum_{j=0}^{n-x} \sum_{k=0}^{b-1}(-1)^{k}\left(\begin{array}{c}x \\ i\end{array}\right)\left(\begin{array}{c}n-x \\ j\end{array}\right)\left(\begin{array}{c}b-1 \\ k\end{array}\right) f^{n-i-j} B(c k+c+i+1, j+1)}{\sum_{i=0}^{x} \sum_{j=0}^{n-x} \sum_{k=0}^{b-1}(-1)^{k}\left(\begin{array}{c}x \\ i\end{array}\right)\left(\begin{array}{c}n-x \\ j\end{array}\right)\left(\begin{array}{c}b-1 \\ k\end{array}\right) f^{n-i-j} B(c(1+k)+i, j+1)}$

As a result, the Bias of $\hat{\pi}_{K W}$ as well as its Mean Square Error is also given by 


$$
\begin{aligned}
& B\left(\hat{\pi}_{K W}\right)=\hat{\pi}_{K W}-\pi \\
& \operatorname{MSE}\left(\hat{\pi}_{K W}\right)=\sum_{x=0}^{n}\left(\hat{\pi}_{K W}-\pi\right)^{2} \phi^{x}(1-\phi)^{n-x}
\end{aligned}
$$

\subsection{Estimation of $\pi$ using the generalised beta prior}

The Generalised Beta prior is defined as

$f(\pi)=\frac{c}{B(a, b)} \pi^{a c-1}\left(1-\pi^{c}\right)^{b-1} ; \quad a, b, c>0$

where $a, b . c$ are the shape parameters of the prior distribution as given in equation (21).

By binomial series expansion, we know that

$\left(1-\pi^{c}\right)^{b-1}=\sum_{k=0}^{b-1}(-1)^{k}\left(\begin{array}{c}b-1 \\ k\end{array}\right)\left(\pi^{c}\right)^{k}$

Consequently

$f(\pi)=\frac{c}{B(a, b)} \sum_{k=0}^{b-1}(-1)^{k}\left(\begin{array}{c}b-1 \\ k\end{array}\right) \pi^{c(k+a)-1}$

As a result, the joint density function of $\pi$ and $\mathrm{X}$ with the generalized beta prior is

$f(X, \pi)=\frac{c\left(\begin{array}{l}n \\ x\end{array}\right)(2 P-1)^{n}}{B(a, b)} \sum_{i=0}^{x} \sum_{j=0}^{n-x} \sum_{k=0}^{b-1}(-1)^{k}\left(\begin{array}{c}x \\ i\end{array}\right)\left(\begin{array}{c}n-x \\ j\end{array}\right)\left(\begin{array}{c}b-1 \\ k\end{array}\right) f^{n-i-j} \pi^{i}(1-\pi)^{j} \pi^{c(k+a)-1}$

The marginal probability density function (pdf) of $X$ can then be obtained from (22) as

$$
\begin{aligned}
& f(X)=\int_{0}^{1} f(X, \pi) d \pi \\
& =D \sum_{i=0}^{x} \sum_{j=0}^{n-x} \sum_{k=0}^{b-1}(-1)^{k}\left(\begin{array}{l}
x \\
i
\end{array}\right)\left(\begin{array}{c}
n-x \\
j
\end{array}\right)\left(\begin{array}{c}
b-1 \\
k
\end{array}\right) f^{n-i-j} B(c(k+a)+i, j+1)
\end{aligned}
$$

where $D=\frac{\left.c_{x}^{n}\right] J(2 p-1)^{n}}{B(a, b)}$

Similarly, we obtained the posterior distribution of $\pi$ given $X$ as

$$
f(\pi \mid X)=\frac{\sum_{i=0}^{x} \sum_{j=0}^{n-x} \sum_{k=0}^{b-1}(-1)^{k}\left(\begin{array}{c}
x \\
i
\end{array}\right)\left(\begin{array}{c}
n-x \\
j
\end{array}\right)\left(\begin{array}{c}
b-1 \\
k
\end{array}\right) f^{n-i-j} \pi^{c(k+a)+i-1}(1-\pi)^{j}}{\sum_{i=0}^{x} \sum_{j=0}^{n-x} \sum_{k=0}^{b-1}(-1)^{k}\left(\begin{array}{c}
x \\
i
\end{array}\right)\left(\begin{array}{c}
n-x \\
j
\end{array}\right)\left(\begin{array}{c}
b-1 \\
k
\end{array}\right) f^{n-i-j} B(c(k+a)+i, j+1)}
$$

In the same way, under the square error loss, the posterior mean which is otherwise known as the Bayes estimator is given by

$$
\hat{\pi}_{G W}=\frac{\sum_{i=0}^{x} \sum_{j=0}^{n-x} \sum_{k=0}^{b-1}(-1)^{k}\left(\begin{array}{c}
x \\
i
\end{array}\right)\left(\begin{array}{c}
n-x \\
j
\end{array}\right)\left(\begin{array}{c}
b-1 \\
k
\end{array}\right) f^{n-i-j} B(c(k+a)+i+1, j+1)}{\sum_{i=0}^{x} \sum_{j=0}^{n-x} \sum_{k=0}^{b-1}(-1)^{k}\left(\begin{array}{c}
x \\
i
\end{array}\right)\left(\begin{array}{c}
n-x \\
j
\end{array}\right)\left(\begin{array}{c}
b-1 \\
k
\end{array}\right) f^{n-i-j} B(c(k+a)+i, j+1)}
$$

The Bias of $\hat{\pi}_{G W}$ and its Mean Square Error (MSE) are respectively given by 


$$
\begin{aligned}
& B\left(\hat{\pi}_{G W}\right)=\hat{\pi}_{G W}-\pi \\
& \operatorname{MSE}\left(\hat{\pi}_{G W}\right)=\sum_{x=0}^{n}\left(\hat{\pi}_{G W}-\pi\right)^{2} \phi^{x}(1-\phi)^{n-x}
\end{aligned}
$$

\section{Results and Discussions}

We wrote suitable codes using R-statistical software to evaluate the derived estimators, bias and mean square errors which are given by equations 18-20 and 2628 at sample sizes 25,100 , and 250 respectively.

From the results presented in Tables and Figs. 1a-6b respectively, when $n=25$, $P=0.1$ and 0.2 , the conventional simple beta estimator is better than the proposed estimators when $\pi$ lies within the range $0.1 \leq \pi<0.7$ while the proposed estimators are better than the conventional simple beta estimator when $\pi$ lies within the range $0.7 \leq \pi<1$. However, the proposed Kumaraswamy beta estimator is the best in obtaining more responses from respondents when $\pi$ lies within the range $0.7 \leq \pi<$ 1.

When $n=100, P=0.1$ and 0.2 , the conventional simple beta estimator is better than the proposed estimators when $\pi$ lies within the range $0.1 \leq \pi \leq 0.5$ while the proposed estimators are better than the conventional simple beta estimator when $\pi$ lies within the range $0.6 \leq \pi<1$. However, the proposed Kumaraswamy beta estimator is the best in obtaining more responses from respondents when $\pi$ lies within the range $0.6 \leq \pi<1$.

When $n=250, P=0.1$ and 0.2 , the conventional simple beta estimator is better than the proposed estimators when $\pi$ lies within the range $0.1 \leq \pi<0.5$ while the proposed estimators are better than the conventional simple beta estimator when $\pi$ lies within the range $0.6 \leq \pi<1$. However, the proposed Kumaraswamy beta estimator is the best in obtaining more responses from respondents when $\pi$ lies within the range $0.6 \leq \pi<1$ respectively.

Table 1a. Mean square errors for Warner [1] RRT at $\mathrm{n}=25, \mathrm{x}=15, \mathrm{P}=0.1$.

\begin{tabular}{lccc}
\hline$\pi$ & MSE BETA & MSE KUMA & MSE GLS \\
\hline 0.1 & $2.593655 \mathrm{E}-09$ & $3.279120 \mathrm{E}-08$ & $3.522085 \mathrm{E}-08$ \\
0.2 & $8.250555 \mathrm{E}-10$ & $2.524261 \mathrm{E}-08$ & $2.737966 \mathrm{E}-08$ \\
0.3 & $4.250544 \mathrm{E}-11$ & $1.868008 \mathrm{E}-08$ & $2.052453 \mathrm{E}-08$ \\
0.4 & $2.460048 \mathrm{E}-10$ & $1.310359 \mathrm{E}-08$ & $1.465545 \mathrm{E}-08$ \\
0.5 & $1.435554 \mathrm{E}-09$ & $8.513156 \mathrm{E}-09$ & $9.772417 \mathrm{E}-09$ \\
0.6 & $3.611152 \mathrm{E}-09$ & $4.908769 \mathrm{E}-09$ & $5.875434 \mathrm{E}-09$ \\
0.7 & $6.772799 \mathrm{E}-09$ & $2.290431 \mathrm{E}-09$ & $2.964500 \mathrm{E}-09$ \\
0.8 & $1.092050 \mathrm{E}-08$ & $6.581424 \mathrm{E}-10$ & $1.039615 \mathrm{E}-09$ \\
0.9 & $1.605424 \mathrm{E}-08$ & $1.190337 \mathrm{E}-11$ & $1.007795 \mathrm{E}-10$ \\
\hline
\end{tabular}

Table 1b. Absolute bias for Warner [1] RRT at $\mathrm{n}=25, \mathrm{x}=15, \mathrm{P}=0.1$.

\begin{tabular}{lccc}
\hline$\pi$ & |BIAS|BETA & |BIAS|KUMA & $\mid$ BIAS| GLS \\
\hline 0.1 & 0.22936215 & 0.81553820 & 0.84521180 \\
0.2 & 0.12936215 & 0.71553820 & 0.74521180 \\
0.3 & 0.02936215 & 0.61553820 & 0.64521180 \\
0.4 & 0.07063785 & 0.51553820 & 0.54521180 \\
\hline
\end{tabular}




\begin{tabular}{lccc}
\hline$\pi$ & $\mid$ BIAS|BETA & |BIAS|KUMA & $\mid$ BIAS| GLS \\
\hline 0.5 & 0.17063785 & 0.41553820 & 0.44521180 \\
0.6 & 0.27063785 & 0.31553820 & 0.34521180 \\
0.7 & 0.37063785 & 0.21553820 & 0.24521180 \\
0.8 & 0.47063785 & 0.11553820 & 0.14521180 \\
0.9 & 0.57063785 & 0.01553820 & 0.04521180 \\
\hline
\end{tabular}

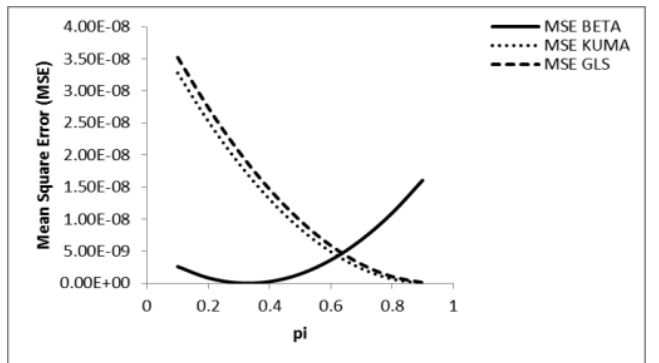

Fig. 1a. Mean square errors for Warner [1] RRT at $\mathrm{n}=25, \mathrm{x}=15, \mathrm{P}=0.1$.

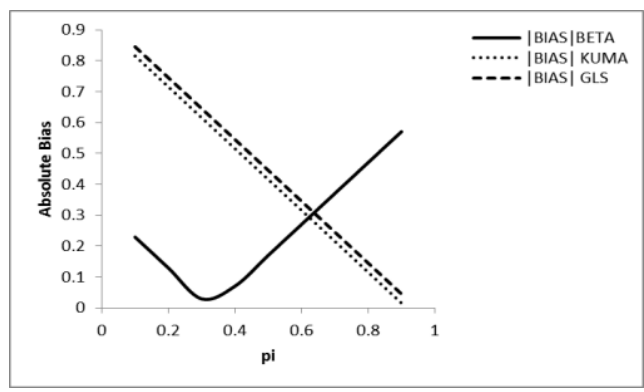

Fig. 1b. Absolute bias for Warner [1] RRT at $\mathrm{n}=25, \mathrm{x}=15, \mathrm{P}=0.1$.

Table 2a. Mean square errors for Warner [1] RRT at $\mathrm{n}=25, \mathrm{x}=15, \mathrm{P}=0.2$.

\begin{tabular}{lccc}
\hline$\pi$ & MSE BETA & MSE KUMA & MSE GLS \\
\hline 0.1 & $2.950359 \mathrm{E}-09$ & $3.194863 \mathrm{E}-08$ & $3.484665 \mathrm{E}-08$ \\
0.2 & $1.031248 \mathrm{E}-09$ & $2.450403 \mathrm{E}-08$ & $2.704986 \mathrm{E}-08$ \\
0.3 & $9.818604 \mathrm{E}-11$ & $1.804548 \mathrm{E}-08$ & $2.023912 \mathrm{E}-08$ \\
0.4 & $1.511737 \mathrm{E}-10$ & $1.257298 \mathrm{E}-08$ & $1.441443 \mathrm{E}-08$ \\
0.5 & $1.190211 \mathrm{E}-09$ & $8.086532 \mathrm{E}-09$ & $9.575786 \mathrm{E}-09$ \\
0.6 & $3.215297 \mathrm{E}-09$ & $4.586132 \mathrm{E}-09$ & $5.723192 \mathrm{E}-09$ \\
0.7 & $6.226433 \mathrm{E}-09$ & $2.071782 \mathrm{E}-09$ & $2.856649 \mathrm{E}-09$ \\
0.8 & $1.022362 \mathrm{E}-08$ & $5.434806 \mathrm{E}-10$ & $9.761541 \mathrm{E}-10$ \\
0.9 & $1.520685 \mathrm{E}-08$ & $1.228796 \mathrm{E}-12$ & $8.170904 \mathrm{E}-11$ \\
\hline
\end{tabular}

Table 2b. Absolute bias for Warner [1] RRT at $\mathrm{n}=25, \mathrm{x}=15, \mathrm{P}=0.2$.

\begin{tabular}{lccc}
\hline$\pi$ & $\mid$ BIAS|BETA & $\mid$ BIAS| KUMA & $\mid$ BIAS| GLS \\
\hline 0.1 & 0.24462626 & 0.80499236 & 0.84070996 \\
0.2 & 0.14462626 & 0.70499236 & 0.74070996 \\
0.3 & 0.04462626 & 0.60499236 & 0.64070996 \\
0.4 & 0.05537374 & 0.50499236 & 0.54070996 \\
0.5 & 0.15537374 & 0.40499236 & 0.44070996 \\
0.6 & 0.25537374 & 0.30499236 & 0.34070996 \\
0.7 & 0.35537374 & 0.20499236 & 0.24070996 \\
0.8 & 0.45537374 & 0.10499236 & 0.14070996 \\
0.9 & 0.55537374 & 0.00499236 & 0.04070996 \\
\hline
\end{tabular}




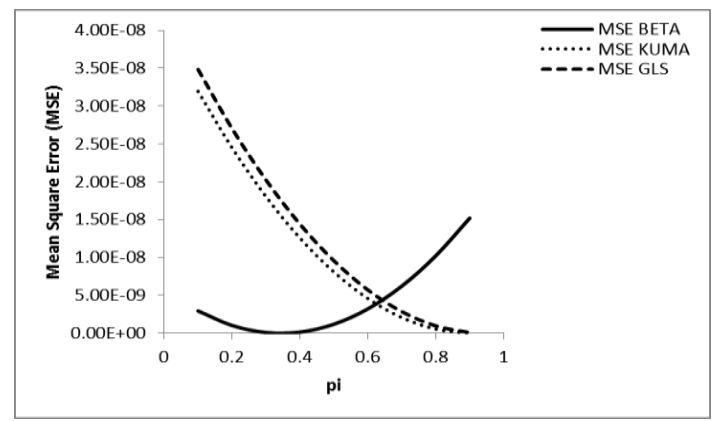

Fig. 2a. Mean square errors for Warner [1] RRT at $\mathrm{n}=25, \mathrm{x}=15, \mathrm{P}=0.2$.

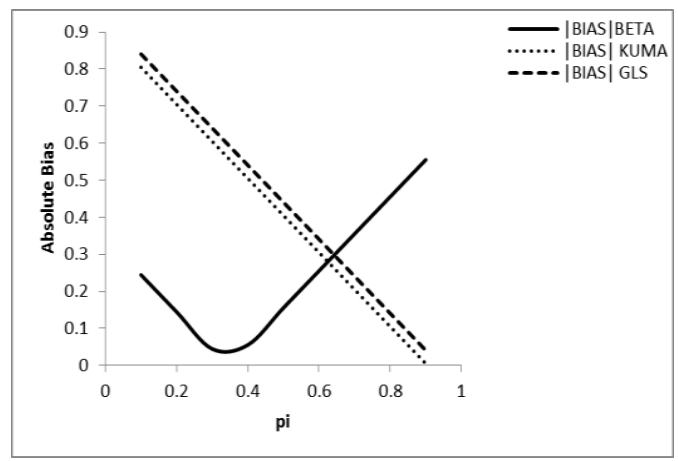

Fig. 2b. Absolute bias for Warner [1] RRT at $\mathrm{n}=25, \mathrm{x}=15, \mathrm{P}=0.2$.

Comment: When $n=25, P=0.1$ and 0.2 , the conventional simple beta estimator is better than the proposed estimators when $\pi$ lies within the range $0.1 \leq \pi<0.7$ while the proposed estimators are better than the conventional simple beta estimator when $\pi$ lies within the range $0.7 \leq \pi<1$. However, the proposed Kumaraswamy beta estimator is the best in obtaining more responses from respondents when $\pi$ lies within the range $0.7 \leq \pi<1$ respectively.

Table 3a. Mean square errors (MSEs) for Warner [1] RRT at $\mathrm{n}=100$, $\mathrm{x}=60, \mathrm{P}=0.1$.

\begin{tabular}{lccc}
\hline$\pi$ & MSE BETA & MSE KUMA & MSE GLS \\
\hline 0.1 & $1.910454 \mathrm{E}-33$ & $4.544300 \mathrm{E}-30$ & $4.571518 \mathrm{E}-30$ \\
0.2 & $3.974625 \mathrm{E}-32$ & $3.567048 \mathrm{E}-30$ & $3.591167 \mathrm{E}-30$ \\
0.3 & $1.957513 \mathrm{E}-31$ & $2.707966 \mathrm{E}-30$ & $2.728985 \mathrm{E}-30$ \\
0.4 & $4.699257 \mathrm{E}-31$ & $1.967052 \mathrm{E}-30$ & $1.984973 \mathrm{E}-30$ \\
0.5 & $8.622694 \mathrm{E}-31$ & $1.344308 \mathrm{E}-30$ & $1.359130 \mathrm{E}-30$ \\
0.6 & $1.372782 \mathrm{E}-30$ & $8.397336 \mathrm{E}-31$ & $8.514565 \mathrm{E}-31$ \\
0.7 & $2.001465 \mathrm{E}-30$ & $4.533281 \mathrm{E}-31$ & $4.619522 \mathrm{E}-31$ \\
0.8 & $2.748316 \mathrm{E}-30$ & $1.850919 \mathrm{E}-31$ & $1.906172 \mathrm{E}-31$ \\
0.9 & $3.613337 \mathrm{E}-30$ & $3.502502 \mathrm{E}-32$ & $3.745154 \mathrm{E}-32$ \\
\hline
\end{tabular}


Table 3b. Absolute bias for Warner [1] RRT at $\mathrm{n}=100, \mathrm{x}=60, \mathrm{P}=0.1$.

\begin{tabular}{lccc}
\hline$\pi$ & BIAS|BETA & BIAS| KUMA & $\mid$ BIAS| GLS \\
\hline 0.1 & 0.01798171 & 0.87699311 & 0.87961547 \\
0.2 & 0.08201829 & 0.77699311 & 0.77961547 \\
0.3 & 0.18201829 & 0.67699311 & 0.67961547 \\
0.4 & 0.28201829 & 0.57699311 & 0.57961547 \\
0.5 & 0.38201829 & 0.47699311 & 0.47961547 \\
0.6 & 0.48201829 & 0.37699311 & 0.37961547 \\
0.7 & 0.58201829 & 0.27699311 & 0.27961547 \\
0.8 & 0.68201829 & 0.17699311 & 0.17961547 \\
0.9 & 0.78201829 & 0.07699311 & 0.07961547 \\
\hline
\end{tabular}

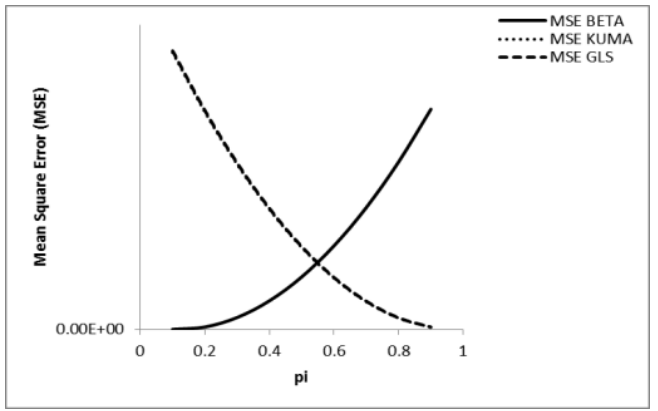

Fig. 3a. Mean square errors for Warner [1] RRT at $\mathrm{n}=100, \mathrm{x}=60, \mathrm{P}=0.1$.

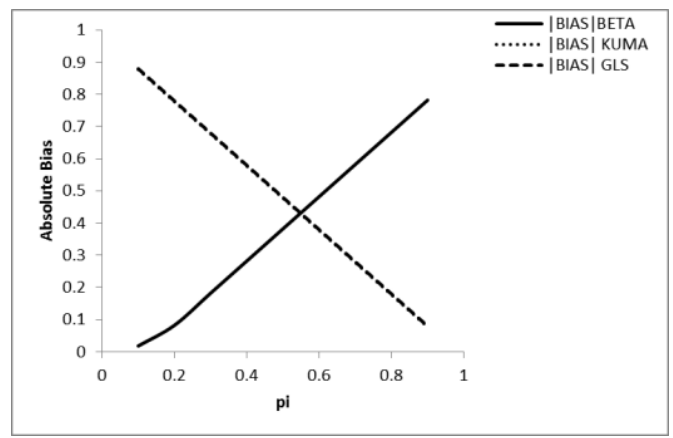

Fig. 3b.Absolute bias for Warner [1] RRT at $\mathrm{n}=100, \mathrm{x}=60, \mathrm{P}=0.1$.

Table 4a. Mean square errors for Warner [1] RRT at $\mathrm{n}=100, \mathrm{x}=60, \mathrm{P}=0.2$.

\begin{tabular}{lccc}
\hline$\pi$ & MSE BETA & MSE KUMA & MSE GLS \\
\hline 0.1 & $1.038804 \mathrm{E}-32$ & $4.497484 \mathrm{E}-30$ & $4.537997 \mathrm{E}-30$ \\
0.2 & $1.992377 \mathrm{E}-32$ & $3.525584 \mathrm{E}-30$ & $3.561464 \mathrm{E}-30$ \\
0.3 & $1.476288 \mathrm{E}-31$ & $2.671854 \mathrm{E}-30$ & $2.703100 \mathrm{E}-30$ \\
0.4 & $3.935031 \mathrm{E}-31$ & $1.936292 \mathrm{E}-30$ & $1.962906 \mathrm{E}-30$ \\
0.5 & $7.575468 \mathrm{E}-31$ & $1.318900 \mathrm{E}-30$ & $1.340881 \mathrm{E}-30$ \\
0.6 & $1.239760 \mathrm{E}-30$ & $8.196778 \mathrm{E}-31$ & $8.370250 \mathrm{E}-31$ \\
0.7 & $1.840142 \mathrm{E}-30$ & $4.386244 \mathrm{E}-31$ & $4.513386 \mathrm{E}-31$ \\
0.8 & $2.558694 \mathrm{E}-30$ & $1.757403 \mathrm{E}-31$ & $1.838214 \mathrm{E}-31$ \\
0.9 & $3.395414 \mathrm{E}-30$ & $3.102549 \mathrm{E}-32$ & $3.447361 \mathrm{E}-32$ \\
\hline
\end{tabular}


Table 4b. Absolute bias for Warner [1] RRT at $\mathrm{n}=100, \mathrm{x}=60, \mathrm{P}=0.2$.

\begin{tabular}{lccc}
\hline$\pi$ & $\mid$ BIAS|BETA & $\mid$ BIAS| KUMA & $\mid$ BIAS| GLS \\
\hline 0.1 & 0.04193045 & 0.87246395 & 0.87638464 \\
0.2 & 0.05806955 & 0.77246395 & 0.77638464 \\
0.3 & 0.15806955 & 0.67246395 & 0.67638464 \\
0.4 & 0.25806955 & 0.57246395 & 0.57638464 \\
0.5 & 0.35806955 & 0.47246395 & 0.47638464 \\
0.6 & 0.45806955 & 0.37246395 & 0.37638464 \\
0.7 & 0.55806955 & 0.27246395 & 0.27638464 \\
0.8 & 0.65806955 & 0.17246395 & 0.17638464 \\
0.9 & 0.75806955 & 0.07246395 & 0.07638464 \\
\hline
\end{tabular}

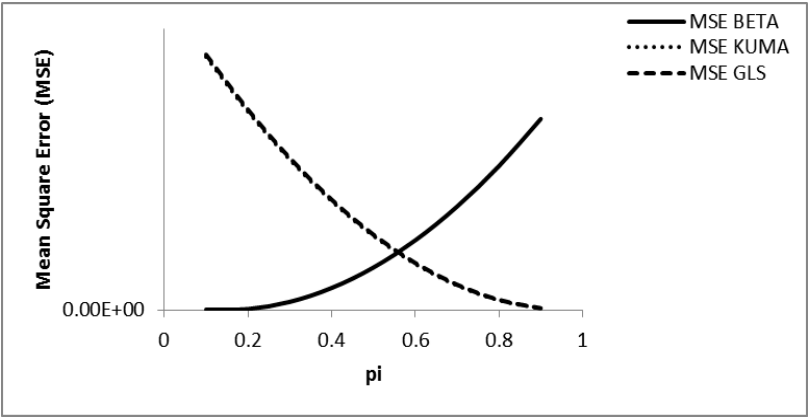

Fig. 4a. Mean square errors for Warner [1] RRT at $\mathrm{n}=100, \mathrm{x}=60, \mathrm{P}=0.2$.

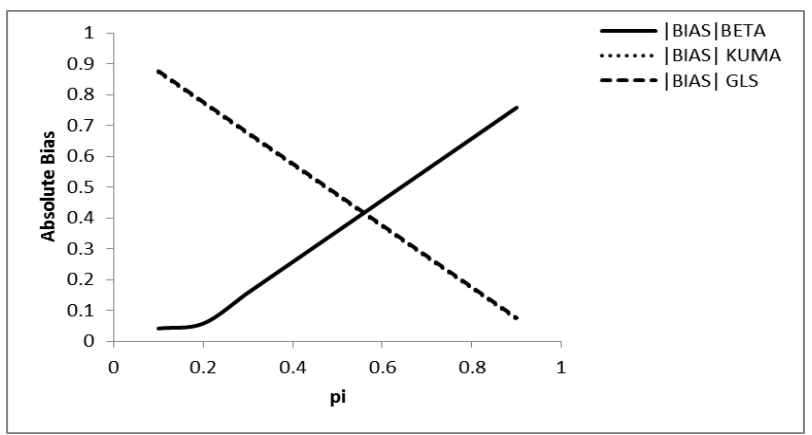

Fig. 4b. Absolute bias for Warner [1] RRT at $\mathrm{n}=100, \mathrm{x}=60, \mathrm{P}=0.2$.

Comment: When $n=100, P=0.1$ and 0.2 , the conventional simple beta estimator is better than the proposed estimators when $\pi$ lies within the range $0.1 \leq \pi$ $\leq 0.5$ while the proposed estimators are better than the conventional simple beta estimator when $\pi$ lies within the range $0.6 \leq \pi<1$. However, the proposed Kumaraswamy beta estimator is the best in obtaining more responses from respondents when $\pi$ lies within the range $0.6 \leq \pi<1$ respectively. 
Table 5a. Mean square errors for Warner [1] RRT at $\mathrm{n}=250, \mathrm{x}=150, \mathrm{P}=0.1$.

\begin{tabular}{lccc}
\hline$\pi$ & MSE BETA & MSE KUMA & MSE GLS \\
\hline 0.1 & $2.391605 E-76$ & $6.732776 \mathrm{E}-74$ & $6.738953 \mathrm{E}-74$ \\
0.2 & $1.988715 \mathrm{E}-75$ & $5.305916 \mathrm{E}-74$ & $5.311400 \mathrm{E}-74$ \\
0.3 & $5.435405 \mathrm{E}-75$ & $4.048770 \mathrm{E}-74$ & $4.053560 \mathrm{E}-74$ \\
0.4 & $1.057923 \mathrm{E}-74$ & $2.961337 \mathrm{E}-74$ & $2.965434 \mathrm{E}-74$ \\
0.5 & $1.742019 \mathrm{E}-74$ & $2.043618 \mathrm{E}-74$ & $2.047022 \mathrm{E}-74$ \\
0.6 & $2.595829 \mathrm{E}-74$ & $1.295612 \mathrm{E}-74$ & $1.298323 \mathrm{E}-74$ \\
0.7 & $3.619353 \mathrm{E}-74$ & $7.173205 \mathrm{E}-75$ & $7.193377 \mathrm{E}-75$ \\
0.8 & $4.812590 \mathrm{E}-74$ & $3.087423 \mathrm{E}-75$ & $3.100662 \mathrm{E}-75$ \\
0.9 & $6.175541 \mathrm{E}-74$ & $6.987772 \mathrm{E}-76$ & $7.050828 \mathrm{E}-76$ \\
\hline
\end{tabular}

Table 5b. Absolute bias for Warner [1] RRT at $\mathrm{n}=250, \mathrm{x}=150, \mathrm{P}=0.1$.

\begin{tabular}{lccc}
\hline$\pi$ & BIAS|BETA & BIAS| KUMA & $\mid$ BIAS| GLS \\
\hline 0.1 & 0.05308861 & 0.89074569 & 0.89115421 \\
0.2 & 0.15308861 & 0.79074569 & 0.79115421 \\
0.3 & 0.25308861 & 0.69074569 & 0.69115421 \\
0.4 & 0.35308861 & 0.59074569 & 0.59115421 \\
0.5 & 0.45308861 & 0.49074569 & 0.49115421 \\
0.6 & 0.55308861 & 0.39074569 & 0.39115421 \\
0.7 & 0.65308861 & 0.29074569 & 0.29115421 \\
0.8 & 0.75308861 & 0.19074569 & 0.19115421 \\
0.9 & 0.85308861 & 0.09074569 & 0.09115421 \\
\hline
\end{tabular}

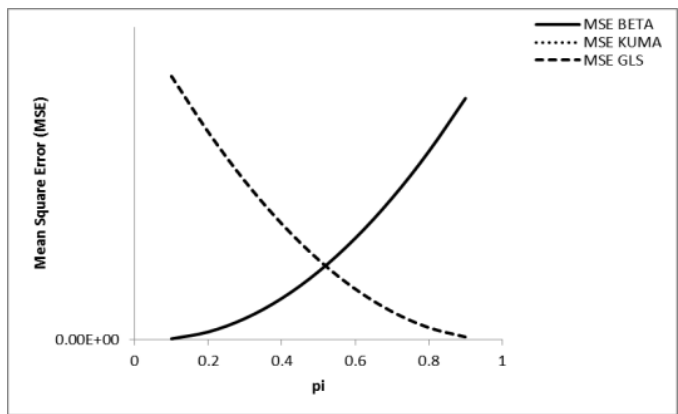

Fig. 5a. Mean square errors for Warner [1] RRT at $\mathrm{n}=250, \mathrm{x}=150, \mathrm{P}=0.1$.

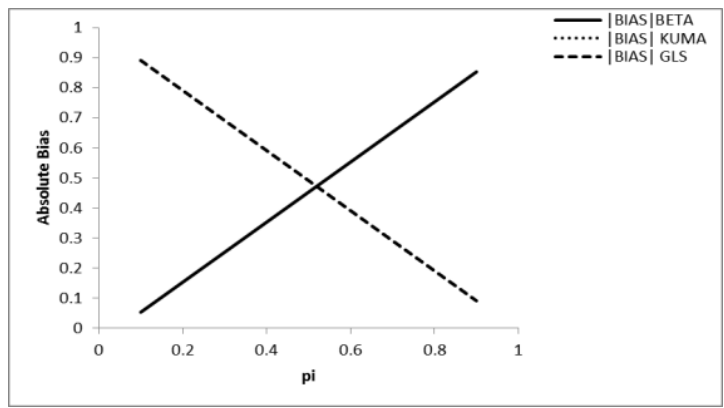

Fig. 5b. Absolute bias for Warner [1] RRT at $\mathrm{n}=250, \mathrm{x}=150, \mathrm{P}=0.1$. 
Table 6a. Mean square errors for Warner [1] RRT at $\mathrm{n}=250, \mathrm{x}=150, \mathrm{P}=0.2$.

\begin{tabular}{lccc}
\hline$\pi$ & MSE BETA & MSE KUMA & MSE GLS \\
\hline 0.1 & $1.701448 \mathrm{E}-76$ & $6.706874 \mathrm{E}-74$ & $6.715781 \mathrm{E}-74$ \\
0.2 & $1.778659 \mathrm{E}-75$ & $5.282925 \mathrm{E}-74$ & $5.290830 \mathrm{E}-74$ \\
0.3 & $5.084309 \mathrm{E}-75$ & $4.028689 \mathrm{E}-74$ & $4.035593 \mathrm{E}-74$ \\
0.4 & $1.008710 \mathrm{E}-74$ & $2.944167 \mathrm{E}-74$ & $2.950069 \mathrm{E}-74$ \\
0.5 & $1.678702 \mathrm{E}-74$ & $2.029359 \mathrm{E}-74$ & $2.034259 \mathrm{E}-74$ \\
0.6 & $2.518408 \mathrm{E}-74$ & $1.284264 \mathrm{E}-74$ & $1.288163 \mathrm{E}-74$ \\
0.7 & $3.527827 \mathrm{E}-74$ & $7.088829 \mathrm{E}-75$ & $7.117805 \mathrm{E}-75$ \\
0.8 & $4.706960 \mathrm{E}-74$ & $3.032154 \mathrm{E}-75$ & $3.051114 \mathrm{E}-75$ \\
0.9 & $6.055807 \mathrm{E}-74$ & $6.726140 \mathrm{E}-76$ & $6.815597 \mathrm{E}-76$ \\
\hline
\end{tabular}

Table 6b. Absolute bias for Warner [1] RRT at $\mathrm{n}=250, \mathrm{x}=150, \mathrm{P}=0.2$.

\begin{tabular}{lccc}
\hline$\pi$ & $\mid$ BIAS|BETA & $\mid$ BIAS| KUMA & $\mid$ BIAS| GLS \\
\hline 0.1 & 0.04477813 & 0.88903066 & 0.88962076 \\
0.2 & 0.14477813 & 0.78903066 & 0.78962076 \\
0.3 & 0.24477813 & 0.68903066 & 0.68962076 \\
0.4 & 0.34477813 & 0.58903066 & 0.58962076 \\
0.5 & 0.44477813 & 0.48903066 & 0.48962076 \\
0.6 & 0.54477813 & 0.38903066 & 0.38962076 \\
0.7 & 0.64477813 & 0.28903066 & 0.28962076 \\
0.8 & 0.74477813 & 0.18903066 & 0.18962076 \\
0.9 & 0.84477813 & 0.08903066 & 0.08962076 \\
\hline
\end{tabular}

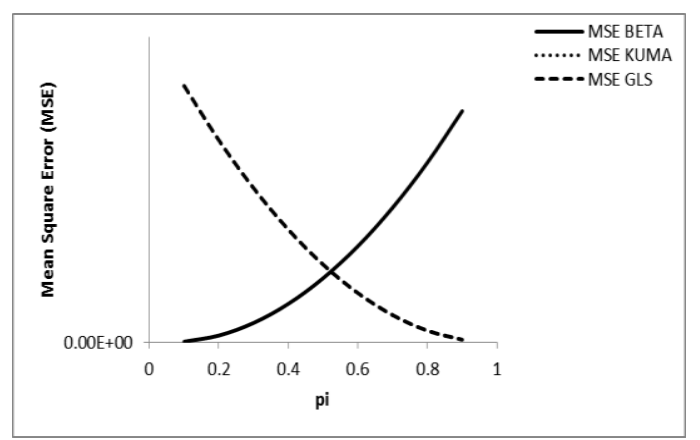

Fig. 6a. Mean square errors for Warner [1] RRT at $\mathrm{n}=250, \mathrm{x}=150, \mathrm{P}=0.2$.

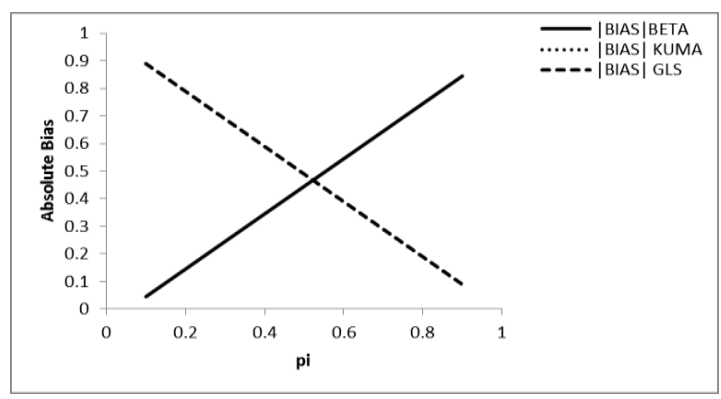

Fig. 6b. Absolute bias for Warner [1] RRT at $\mathrm{n}=250, \mathrm{x}=150, \mathrm{P}=0.2$. 
Comment: When $n=250, P=0.1$ and 0.2 , the conventional simple beta estimator is better than the proposed estimators when $\pi$ lies within the range $0.1 \leq \pi$ $<0.5$ while the proposed estimators are better than the conventional simple beta estimator when $\pi$ lies within the range $0.6 \leq \pi<1$. However, the proposed Kumaraswamy beta estimator is the best in obtaining more responses from respondents when $\pi$ lies within the range $0.6 \leq \pi<1$ respectively.

\section{Conclusion}

We have proposed the alternative Bayesian estimators of the population proportion when life data were gathered through the administration of questionnaires on abortion on 300 matured women in addition to the conventional simple beta estimator proposed by Winkler and Franklin [10]. We observed clearly from the results that for relatively small, intermediate as well as large sample sizes, the proposed Bayesian estimators are more sensitive in capturing sensitive attribute than the conventional simple beta estimator. In particular, the proposed Kumaraswamy beta estimator is the best in obtaining information from respondents in survey which asks sensitive questions.

\section{References}

1. S. L. Warner, J. Am. Statist. Assoc. 60, 63 (1965). http://dx.doi.org/10.1080/01621459.1965.10480775

2. B. Greenberg, A. Abul-Ela, W. Simmons, and D. Horvitz, J. Am. Statist. Assoc. 64, 529 (1969).

3. N. S. Mangat and R. Singh, Biometrika. 77, 439 (1990). http://dx.doi.org/10.1093/biomet/77.2.439

4. N. S. Mangat, J. Roy. Statist. Soc. Ser. B, 56(1), 93 (1994).

5. T. C. Christofides, Metrika 57, 195 (2003). http://dx.doi.org/10.1007/s001840200216

6. J. M. Kim and D. W. Warde, J. Statist. Plann. Inference 120(1-2), 155 (2004). http://dx.doi.org/10.1016/S0378-3758(02)00500-1

7. F. B. Adebola and A. O. Adepetun, J. Nigerian Assoc. Math. Phys. 19, 119 (2011).

8. F. B. Adebola and A. O. Adepetun, Int. J. Math. Theor. Model. 2, 61 (2012).

9. A. O. Adepetun and F. B. Adebola, Int. J. Math. Theor. Model. 4, 58 (2014).

10. R. Winkler and L. Franklin, J. Amer. Statist. Assoc. 74, 207 (1979). http://dx.doi.org/10.1080/01621459.1979.10481639

11. G. Pitz, J. Psychological Bull. 87, 209 (1980). http://dx.doi.org/10.1037/00332909.87.1.209

12. A. O’Hagan, J. Am. Statist. Assoc. 82, 580 (1987). http://dx.doi.org/10.1080/01621459.1987.10478469

13. M. Oh, J. Kor. Statist. Soc. 23, 463 (1994).

14. H. Migon and V. Tachibana, Comput. Statist. Data Analysis 24, 401 (1997). http://dx.doi.org/10.1016/S0167-9473(96)00075-8

15. N. Unnikrishnan and S. Kunte, Sankhya B 61, 422 (1999).

16. J. M. Kim, J. M. Tebbs, and S. W. An, J. Statist. Plann. Inference 36(4), 1554 (2006). http://dx.doi.org/10.1016/i.jspi.2004.10.005

17. Z. Hussain and J. Shabbir, Pak. J. Statist. 25(1), 27 (2009).

18. Z. Hussain and J. Shabbir, J. Probability Statist. Sci. 7(1), 71 (2009). 
19. Z. Hussain and J. Shabbir, Electronic J. Appl. Statist. Analysis 5(2), 213 (2012).

20. A. O. Adepetun and A. A. Adewara, Amer. J. Comput. Appl. Math. 4(4), 130 (2014).

21. A. O. Adepetun and A. A. Adewara, Int. J. Scient. Eng. Res. 6(2), 1634 (2015).

22. A. O. Adepetun and A. A. Adewara, Punjab Univ. J. Math. 48(1), 29 (2016). 